TITLE:

\title{
Vibrational spectroscopy and relaxation of an anharmonic oscillator coupled to harmonic bath.
}

\author{
$\operatorname{AUTHOR(S):~}$ \\ Joutsuka, Tatsuya; Ando, Koji
}

\section{CITATION:}

Joutsuka, Tatsuya ...[et al]. Vibrational spectroscopy and relaxation of an anharmonic oscillator coupled to harmonic bath.. The Journal of chemical physics 2011, 134(20): 204511.

ISSUE DATE:

2011-05-28

URL:

http://hdl.handle.net/2433/141992

RIGHT:

(C) 2011 American Institute of Physics 


\title{
Vibrational spectroscopy and relaxation of an anharmonic oscillator coupled to harmonic bath
}

\author{
Tatsuya Joutsuka a) and Koji Ando \\ Department of Chemistry, Kyoto University, Sakyo-ku, Kyoto 606-8502, Japan
}

(Received 5 March 2011; accepted 5 May 2011; published online 31 May 2011)

\begin{abstract}
The vibrational spectroscopy and relaxation of an anharmonic oscillator coupled to a harmonic bath are examined to assess the applicability of the time correlation function (TCF), the response function, and the semiclassical frequency modulation (SFM) model to the calculation of infrared (IR) spectra. These three approaches are often used in connection with the molecular dynamics simulations but have not been compared in detail. We also analyze the vibrational energy relaxation (VER), which determines the line shape and is itself a pivotal process in energy transport. The IR spectra and VER are calculated using the generalized Langevin equation (GLE), the Gaussian wavepacket (GWP) method, and the quantum master equation (QME). By calculating the vibrational frequency TCF, a detailed analysis of the frequency fluctuation and correlation time of the model is provided. The peak amplitude and width in the IR spectra calculated by the GLE with the harmonic quantum correction are shown to agree well with those by the QME though the vibrational frequency is generally overestimated. The GWP method improves the peak position by considering the zero-point energy and the anharmonicity although the red-shift slightly overshoots the QME reference. The GWP also yields an extra peak in the higher-frequency region than the fundamental transition arising from the difference frequency of the center and width oscillations of a wavepacket. The SFM approach underestimates the peak amplitude of the IR spectra but well reproduces the peak width. Further, the dependence of the VER rate on the strength of an excitation pulse is discussed. () 2011 American Institute of Physics. [doi:10.1063/1.3594093]
\end{abstract}

\section{INTRODUCTION}

Vibrational relaxation (VR) plays an essential role in many chemical processes in liquids, and the interpretation of the coherence relaxation (dephasing) and vibrational energy relaxation (VER) ${ }^{1,2}$ is vital to reveal the dynamic mechanisms of proton transfer, ${ }^{3-5}$ energy transport, ${ }^{6,7}$ and other chemical processes. Especially, recent advances in ultrafast spectroscopy have clarified dynamical aspects of VR in liquids. ${ }^{8-10}$ Here, the hydrogen bond among molecules or between molecules and ions is so strong that the potential of a local oscillator (e.g., $\mathrm{OH}$ stretch) is strongly anharmonic. In particular, infrared (IR) spectroscopy is one of the ideal techniques to probe their dynamics, because the instantaneous frequency of the light-absorbing local oscillator (e.g., an $\mathrm{OH}$ stretch of an HOD molecule) is closely correlated to its local environment (e.g., the strength of the hydrogen bond). Comparison of molecular simulation results with experimental IR spectra can elucidate a molecular-level VR mechanism.

In comparing simulation results with experimental IR spectra, it is sometimes difficult to analyze the IR spectra on the basis of the normal mode ${ }^{11}$ analysis owing to strong anharmonicity as encountered in liquids, especially in aqueous solutions. To overcome the difficulty, three major approaches are often employed: ${ }^{12}$ 1. time correlation function (TCF), 2. response function (RF), and 3. semiclassical frequency modulation (SFM) model. ${ }^{10,13-18}$ The first approach is based on

\footnotetext{
a) Author to whom correspondence should be addressed. Electronic mail: joutsuka@kuchem.kyoto-u.ac.jp.
}

the linear response theory and usually utilizes the quantum correction factor (QCF) to approximate the quantum TCF by the classical TCF. One advantage of this approach is that the classical TCF can be computed straightforwardly via classical molecular dynamics (MD) simulation. With this method, one commonly compares the simulated IR spectra with the experimental ones in liquids (e.g., liquid water ${ }^{19}$ ). The second approach has the advantage that the linear response theory need not be assumed and the non-linear responses are effectively included. The third approach approximates the dipole TCF in the IR absorption coefficient semiclassically in terms of the excitation frequency modulation. Here, the Condon approximation and cumulant expansion are commonly used. This approach works well for molecular systems where the dominant relaxation processes are bath-induced vibrational dephasing and reorientational dynamics. ${ }^{8}$ The validity of this approach has been examined for an anharmonic diatomic molecule imbedded in Lennard-Jones fluid. ${ }^{20}$ In comparison, much less has been studied about the validity of the TCF and SFM approaches for liquids, especially for aqueous solutions. This validation is essential to compare simulation and experiment. Another advantage of the third approach is that the dipole TCF can be rewritten in the form of the frequency TCF (FTCF). Therefore, it has been adapted for discussing (in)homogeneous line broadening, ${ }^{21}$ which offers deep understanding of spectroscopy through FTCFs and for comparing simulated and experimental IR spectra.

Accordingly, the purpose of this paper is to assess the applicability of the above-mentioned three approaches to 
calculate IR spectra. We also evaluate the VER rate, which influences the IR line shape and is an essential process itself in energy transport. We employ the Morse potential for an anharmonic potential of molecular vibrations. The parameters are chosen to have relevance to the aqueous solution systems such as $\mathrm{CN}^{-} / \mathrm{H}_{2} \mathrm{O},{ }^{17,22} \mathrm{HOD} / \mathrm{D}_{2} \mathrm{O},{ }^{10,23} \mathrm{OD}^{-} / \mathrm{HOD} / \mathrm{D}_{2} \mathrm{O},{ }^{9}$ and so on; however, our primary aim is to gain insight into various theoretical approaches rather than to make detailed comparison with specific systems.

In modeling VR, we employ the system-bath Hamiltonian, which involves separating the Hamiltonian into a system part (the vibrational mode of interest), a bath (reservoir) part, and the system-bath interaction. Because of the simplicity of this model, the quantum-mechanical density matrix formalism, such as the quantum master equation (QME) ${ }^{24,25}$ can be employed. Finding the FTCF starting from the system-bath model will offer an insight into whether the line shape is homogeneous or inhomogeneous in the theoretical bath model through FTCFs. In the course of the analysis, we also validate the adequacy of the QCF and the Gaussian wavepacket (GWP) method. Furthermore, to analyze the effect of anharmonicity of molecular vibrations, the excitation energy dependence of the VER rate is examined.

The outline of the remainder of this paper is as follows. In Sec. II, we will begin by summarizing the backgrounds of TCF (Sec. II A), RF (Sec. II B), and SFM (Sec. II C) approaches. In Sec. II C, the perturbational calculation of frequency shift is also described in connection with the SFM. We summarize the simulation techniques in Sec. III. The generalized Langevin equation (GLE), the GWP method, and the QME are described in Secs. III A, III B, and III C, respectively. In Secs. IV and V, the computational details and results are described. The FTCF, IR spectra, and anharmonicity of our model are discussed in Secs. V A, V B, and V C. An extra peak in the GWP method and motional narrowing from the SFM are analyzed in Secs. V D and V E. The energy relaxation time is detailed in Sec. V F. In Sec. VI, we conclude.

\section{THEORETICAL BACKGROUND}

\section{A. Time-correlation function}

The IR absorption coefficient per unit length is given by the Fourier transform of the dipole TCF (Ref. 26)

$$
\alpha(\omega)=\frac{4 \pi^{2} \omega}{3 V \hbar c n(\omega)}\left(1-e^{-\beta \hbar \omega}\right) I(\omega),
$$

where $V$ is the volume of the system with the refractive index $n(\omega)$ at frequency $\omega, c$ is the speed of light, and $\beta=1 / k_{\mathrm{B}} T$, where $k_{\mathrm{B}}$ is the Boltzmann constant and $T$ is the temperature. $I(\omega)$ is defined by

$$
I(\omega)=\frac{1}{2 \pi} \int_{-\infty}^{\infty} d t e^{-i \omega t}\langle M(0) M(t)\rangle,
$$

in which $M$ is the dipole moment operator. Usually, $\alpha(\omega)$ is decomposed with the QCF $Q_{\mathrm{QC}}(\omega)$

$$
\alpha(\omega)=\frac{4 \pi^{2} \omega}{3 V \hbar c n(\omega)}\left(1-e^{-\beta \hbar \omega}\right) Q_{\mathrm{QC}}(\omega) I_{\mathrm{cl}}(\omega),
$$

in which $I_{\mathrm{cl}}(\omega)$ is the classical mechanical analogue of $I(\omega)$ in Eq. (2) that can be computed from the classical TCF of $M$. In this paper, we use only the harmonic QCF: ${ }^{27,28}$

$$
Q_{\mathrm{QC}}(\omega)=\frac{\beta \hbar \omega}{1-e^{-\beta \hbar \omega}},
$$

which is usually better than the other QCFs to calculate IR spectra. ${ }^{26}$ If the bath is harmonic and the force is linear in the bath coordinates, then the harmonic QCF is exact. Otherwise, the harmonic QCF sometimes leads to the underestimation of the amplitudes of IR spectra, and, therefore, other QCFs have been proposed. ${ }^{10,27,28}$ For instance, the Schofield $\mathrm{QCF} e^{\beta \hbar \omega / 2}$ is much larger than the harmonic one for the highfrequency oscillation $(\beta \hbar \omega \gg 1)$ and suitable when the harmonic one is too small. On the other hand, the standard QCF $2 /\{1+\exp (-\beta \hbar \omega)\}$ is much smaller than the harmonic one for $\beta \hbar \omega \gg 1$.

\section{B. Response function}

The absorption coefficient in Eq. (1) can be rewritten as ${ }^{12}$

$$
\alpha(\omega)=\frac{4 \pi^{2} \omega}{3 V \hbar c n(\omega)} \frac{1}{2 \pi \beta \omega} \int_{-\infty}^{\infty} d t e^{-i \omega t} S(t),
$$

with the linear RF

$$
S(t)=\frac{i}{\hbar} \theta(t)\left\langle[M(t), M(0)] \rho_{\mathrm{eq}}\right\rangle .
$$

Here, we have applied the perturbation $-E(t) M$ at $t=0$ to the equilibrium density operator $\rho_{\text {eq }}$ with the electric field $E(t)$ and $\theta(t)$ is the Heavyside step function.

\section{Semiclassical frequency modulation model}

We now approximate Eq. (2) to calculate IR spectra for a fundamental $0-1$ transition of a vibrational mode. Throughout this paper, we assume that the transition dipole operator is independent of the bath coordinates (the Condon approximation). Moreover, if we consider vibrational anharmonicity in dephasing time calculations by the SFM, then $I(\omega)$ is rewritten by ${ }^{10,12-18,20,29}$

$$
\begin{aligned}
I(\omega)= & \frac{\left|M_{10}\right|^{2}}{2 \pi} \int_{-\infty}^{\infty} d t e^{i\left(\omega-\left\langle\omega_{10}\right\rangle\right) t-t /\left(2 T_{1}\right)} \\
& \times\left\langle\exp \left[i \int_{0}^{t} d \tau \delta \omega_{10}(\tau)\right]\right\rangle,
\end{aligned}
$$

where $\omega_{10}(t)$ is the instantaneous transition frequency between the ground and excited states. $\delta \omega_{10}(t)=\omega_{10}(t)-\left\langle\omega_{10}\right\rangle$ is the instantaneous frequency shift from the mean value $\left\langle\omega_{10}\right\rangle$ induced by the coupling to the bath. This can approximately be calculated by

$$
\hbar \delta \omega_{10}(t)=\left(Q_{11}-Q_{00}\right) F_{1} .
$$

$F_{1}$ is (minus) the force exerted by the solvent on the fixed oscillator coordinate at $Q=0$, and $Q_{00}$ and $Q_{11}$ are the matrix elements of the position operator. This is the first-order perturbational approximation and the higher-order terms vanish if the higher-order derivatives of the system-bath coupling with respect to the oscillator coordinate are absent. One of the 
advantages of this method is a direct mapping of a bath configuration to the instantaneous frequency shift. Given a bath configuration, $F_{1}$ is calculated, and then $\delta \omega_{10}(t)$ can unambiguously be determined by Eq. (8). This property enables us to focus on the estimation of the error in the SFM approach in Eq. (7).

In this approach, we assume that the molecular vibration of interest is adiabatic. In other words, the oscillation period is short compared to time scales of bath motions. This corresponds to ignoring population relaxation. If the population lifetime is comparable to the dephasing time, the population relaxation should be considered in some way. Therefore, the effects of population relaxation are included with a phenomenological exponential damping constant $T_{1}$. For a harmonic potential, the frequency fluctuation vanishes and the line shape is determined only by the lifetime broadening. For an anharmonic potential, on the other hand, the line shape is broadened also by the frequency fluctuation. Equation (8) works well if the bath correlation time is much shorter than the population relaxation time, but otherwise the correlation between phase and population relaxations would become nonnegligible. Therefore, we evaluate the applicability of Eq. (8) in this paper.

Moreover, by combining Eq. (7) with the cumulant expansion, $I(\omega)$ can be rewritten as

$$
I(\omega)=\frac{\left|M_{10}\right|^{2}}{2 \pi} \int_{-\infty}^{\infty} d t e^{i(\omega-\langle\omega\rangle) t-t /\left(2 T_{1}\right)} e^{-g(t)},
$$

where

$$
g(t)=\int_{0}^{t} d \tau(t-\tau) C(\tau)
$$

and $C(t)$ is the (complex) quantum FTCF $C(t) \equiv$ $\langle\delta \omega(t) \delta \omega(0)\rangle$. In this paper, this is approximated by the classical FTCF. The cumulant expansion is accurate when the bath fluctuation can be considered as a Gaussian process.

\section{SIMULATION TECHNIQUES}

We employ the system-bath Hamiltonian of the form

$$
H=\frac{P^{2}}{2 \mu}+V(Q)+\sum_{i}\left\{\frac{p_{i}^{2}}{2 m_{i}}+\frac{m_{i}}{2} \omega_{i}^{2}\left(q_{i}-\frac{c_{i} V_{\mathrm{S}}}{m_{i} \omega_{i}^{2}}\right)^{2}\right\}
$$

where $Q, P, \mu$, and $V(Q)$ are the coordinate, conjugate momentum, effective mass, and potential of the system oscillator, respectively. $q_{i}, p_{i}, m_{i}$, and $\omega_{i}$ are the coordinate, conjugate momentum, mass, and frequency of the $i$ th bath oscillator, respectively. In addition, $V_{\mathrm{S}}$ is assumed to be a linear operator of the system, $V_{\mathrm{S}}=Q$ in this paper. $c_{i}$ denotes the coupling constant to the $i$ th bath oscillator. One of the advantages of this Hamiltonian is that the higher-order terms in Eq. (8) vanish because the higher-order derivatives of the system-bath coupling with respect to the oscillator coordinate are absent. Thus, the expression of the instantaneous frequency shift in Eq. (8) is simple. Connection of the model to more realistic systems can be made by combining with MD simulations, where the bath parameters are evaluated from the Fourier transform of a certain force-force TCF. This methodology has been applied previously to several systems. ${ }^{30,31}$ In this work, however, we employ simple spectral density [Eq. (15) below] to compare various theoretical strategies.

\section{A. Generalized Langevin equation}

From Eq. (11), the generalized Langevin equation is derived as ${ }^{24}$

$$
\mu \ddot{Q}=-\frac{d V(Q)}{d Q}-\mu \int_{0}^{t} Z(t-\tau) \dot{Q}(\tau) d \tau+R(t),
$$

where $Z(t)$ is the friction kernel

$$
Z(t)=\frac{2}{\pi \mu} \int_{0}^{\infty} d \omega \frac{J(\omega)}{\omega} \cos \omega t
$$

and $J(\omega)$ is the spectral density

$$
J(\omega)=\frac{\pi}{2} \sum_{j} \frac{c_{j}^{2}}{m_{j} \omega_{j}} \delta\left(\omega-\omega_{j}\right) .
$$

and $R(t)$ is the random force. In this paper, we assume the Drude spectral density

$$
J(\omega)=\frac{\mu \zeta \omega \gamma^{2}}{\omega^{2}+\gamma^{2}}
$$

giving $Z(t)=\zeta \gamma e^{-\gamma t}$. The Landau-Teller (LT) formula for the energy relaxation time $T_{1}$ is given by ${ }^{20}$

$$
\frac{1}{T_{1}}=Z^{\prime}(\omega)=\frac{\zeta \gamma^{2}}{\omega^{2}+\gamma^{2}},
$$

in which $Z^{\prime}(\omega)$ is the real part of the Fourier-Laplace transform of $Z(t)$. Since this formula is accurate for a harmonic potential with a bilinear system-bath coupling, ${ }^{20,32}$ we examine the accuracy for an anharmonic potential with a moderate coupling to the bath in this paper.

For the spectral density in Eq. (15), it is possible to rewrite Eq. (12) in an equivalent Markovian form by introducing an auxiliary momentum of the bath $s^{33,34}$

$$
\dot{P}=-\frac{d V(Q)}{d Q}+\sqrt{\zeta \gamma} s
$$

and

$$
\dot{s}=-\sqrt{\zeta \gamma} P-\gamma s+r(t) .
$$

Here, $r$ is a white noise, with $\langle r(t) r(0)\rangle=2 \gamma \mu k_{\mathrm{B}} T \delta(t)$. The second term in Eq. (17) can be considered as the force acting onto the system oscillator by the auxiliary momentum. Equations (17) and (18) are used because of the computational efficiency, which arises from the absence of the convolution and the generation of the colored random force in Eq. (12).

\section{B. Gaussian wavepacket method}

In this section, we summarize the GWP method. ${ }^{19,35,36}$ This method is similar to the thawed GWP method m, $38^{37}$ and the single-configuration version of Gaussian-based multiconfiguration time-dependent Hartree (G-MCTDH) method. ${ }^{39,40}$ A particular feature is the extended Hamiltonian formalism (Eqs. (21)-(24)) that enables straightforward derivation of the semiquantal GLE (Ref. 35) (Eqs. (25) and (26)). This aspect 
is also shared by the expectation-value approaches such as the second-order quantum Hamilton dynamics ${ }^{41,42}$ and quantal cumulant dynamics. ${ }^{43,44}$

For simplicity of notation, we work in this section with $\hbar=1$ and assume that the coordinates are mass scaled. Here, we invoke the time-dependent Hartree approximation with the squeezed coherent state Gaussian basis functions,

$$
\begin{aligned}
\Psi(\mathbf{q}, t)= & \prod_{\alpha=1}^{f} N_{\alpha} \exp \left\{A_{\alpha}(t)\left[q_{\alpha}-Q_{\alpha}(t)\right]^{2}\right. \\
& \left.+i P_{\alpha}(t)\left[q_{\alpha}-Q_{\alpha}(t)\right]\right\}
\end{aligned}
$$

where $f$ is the degrees of freedom, $N_{\alpha}=\left(2 \pi \rho_{\alpha}(t)^{2}\right)^{-1 / 4}$ is the normalization factor, and

$$
A_{\alpha}(t)=\frac{-1+2 i \rho_{\alpha}(t) \pi_{\alpha}(t)}{4 \rho_{\alpha}(t)^{2}},
$$

which is specified by the time-dependent parameters $\left\{Q_{\alpha}(t), P_{\alpha}(t), \rho_{\alpha}(t), \pi_{\alpha}(t)\right\}$ to be determined from the timedependent variational principle (TDVP). The time-dependent parameters $Q_{\alpha}(t)$ and $\rho_{\alpha}(t)$ describe the centers and widths of wavepackets. The parameters $P_{\alpha}(t)$ and $\pi_{\alpha}(t)$ are the conjugate momenta of $Q_{\alpha}(t)$ and $\rho_{\alpha}(t)$, respectively. A set of equations of motion for these parameters are obtained by the TDVP for the parameters in Eq. (19). The resulting equations of motions of the classical Hamilton form

$$
\begin{gathered}
\dot{Q}_{\alpha}=\partial H_{\mathrm{ext}} / \partial P_{\alpha}, \quad \dot{P}_{\alpha}=-\partial H_{\mathrm{ext}} / \partial Q_{\alpha}, \\
\dot{\rho}_{\alpha}=\partial H_{\mathrm{ext}} / \partial \pi_{\alpha}, \quad \dot{\pi}_{\alpha}=-\partial H_{\mathrm{ext}} / \partial \rho_{\alpha}
\end{gathered}
$$

are expressed by the extended Hamiltonian

$$
H_{\mathrm{ext}}=\sum_{\alpha=1}^{f}\left[\frac{P_{\alpha}^{2}}{2}+\frac{\pi_{\alpha}^{2}}{2}\right]+V_{\mathrm{ext}},
$$

with the extended potential

$$
V_{\mathrm{ext}}=\sum_{\alpha=1}^{f} \frac{1}{8 \rho_{\alpha}^{2}}+\langle\Psi|V| \Psi\rangle=\sum_{\alpha=1}^{f} \frac{1}{8 \rho_{\alpha}^{2}}+V(\boldsymbol{Q}, \boldsymbol{\rho}) .
$$

Here, $V(\boldsymbol{Q})$ is averaged by the GWP in Eq. (19) to yield $V(\boldsymbol{Q}, \boldsymbol{\rho})$. This extended potential can be derived exactly for the Morse potential and, therefore, no approximation is needed in its calculation. The GWP method can be directly applied to the GLE, ${ }^{35}$ yielding

$$
\begin{gathered}
\mu \ddot{Q}=-\frac{d V_{\mathrm{ext}}(Q, \rho)}{d Q}-\mu \int_{0}^{t} Z(t-\tau) \dot{Q}(\tau) d \tau+R(t) \\
\mu \ddot{\rho}=-\frac{d V_{\mathrm{ext}}(Q, \rho)}{d \rho} .
\end{gathered}
$$

Here, the mass $\mu$ and $\hbar$ have been retrieved. It is noted that there is no friction term in Eq. (26). This is a particular consequence of the bilinear coupling model $\left(V_{\mathrm{S}}=Q\right.$ in Eq. (11)). It has been shown ${ }^{35}$ that inclusion of higher-order couplings yields the friction term in the equation of motion for $\rho$.

\section{Time-local quantum master equation}

The time-local (convolution-less) QME in the energy representation is expressed as ${ }^{25,45-50}$

$$
\begin{aligned}
\frac{\partial}{\partial t} \rho_{a b}(t)= & -i \omega_{a b} \rho_{a b}(t)-\frac{i}{\hbar} \sum_{c}\left(\bar{V}_{a c} \rho_{c b}-\rho_{a c} \bar{V}_{c b}\right) \\
& +\sum_{c, d} R_{a b, c d}(t) \rho_{c d}(t),
\end{aligned}
$$

where

$$
\begin{aligned}
R_{a b, c d}(t)= & \Gamma_{d b, a c}\left(\omega_{c a} ; t\right)+\Gamma_{c a, b d}^{*}\left(\omega_{d b} ; t\right) \\
& -\delta_{b d} \sum_{e} \Gamma_{a e, e c}\left(\omega_{c e} ; t\right) \\
& -\delta_{a c} \sum_{e} \Gamma_{b e, e d}^{*}\left(\omega_{d e} ; t\right),
\end{aligned}
$$

and

$$
\Gamma_{a b, c d}\left(\omega_{d c} ; t\right)=\frac{V_{a b} V_{c d}}{\hbar^{2}} \int_{0}^{t} d s C_{\mathrm{B}}(s) e^{-i \omega_{d c} s} .
$$

Here, $a, b, c$, and $d$ are the vibrational eigenstates of the system potential $V(Q)$, and $V_{a b}=\left\langle a\left|V_{\mathrm{S}}\right| b\right\rangle$. The second term in Eq. (27) describes the renormalization term and $\bar{V}=$ $\mu Z(0) V_{\mathrm{S}}^{2} / 2$. The bath TCF is expressed as

$$
C_{\mathrm{B}}(t)=\frac{\hbar}{\pi} \int_{0}^{\infty} d \omega J(\omega)\left[\left(n_{\mathrm{B}}(\omega)+1\right) e^{-i \omega t}+n_{\mathrm{B}}(\omega) e^{i \omega t}\right]
$$

with $n_{\mathrm{B}}(\omega)=1 /\left(e^{\beta \hbar \omega}-1\right)$. $R_{a b, c d}(t)$ can be evaluated analytically for the spectral density in Eq. (15) and, therefore, Eq. (27) can be solved quite effectively.

The QME is exact when the system Hamiltonian commutes with the system-bath coupling Hamiltonian (pure dephasing). ${ }^{47,49,50}$ For other cases including ours, it involves standard approximations such as the time-local deconvolution and the second-order perturbative truncation for the systembath coupling, so that it is well expected to provide reliable reference for dynamics near equilibrium. Indeed, the validity was demonstrated by a similar model for linear IR spectra by comparing to the exact simulation result ${ }^{47}$ (whereas a possible cancellation of errors was also suggested ${ }^{49}$ ). Moreover, the VERs calculated by the GLE and QME for a harmonic potential agree well with each other as shown in Fig. S1. ${ }^{51}$ This agreement is justified by the analytical result by Bader et al. ${ }^{27}$ which states that the VER rate for a harmonic quantum mechanical system coupled to a harmonic bath is the same as that for a classical one.

\section{COMPUTATIONAL DETAILS}

In this section, we describe the computational details on the calculation of IR spectra and VER rates. To focus on the adequacy of the approximations, we employ the Condon approximation (the transition dipole moment independent of the bath coordinates), although the non-Condon effect is often important to interpret the IR spectra of molecules. ${ }^{29}$ To be more precise, the dipole moment operator is approximated as 
$M=e Q$, where $e(>0)$ is the unit charge. The effects of rotations to the spectra are also neglected in this study, which was discussed elsewhere. ${ }^{52}$

Throughout this paper, the Morse potential $V(Q)=$ $D\left(1-e^{-\alpha Q}\right)^{2}$ is chosen as an anharmonic model potential to represent the potential energy of molecules in liquids. Since aqueous solutions at ambient conditions are of our particular interest, the simulations were performed for a particle with the mass of a hydrogen atom at a temperature of $300 \mathrm{~K}$ under $V(Q)$ corresponding to the typical $\mathrm{OH}$ stretching vibrations. The Morse parameters were thus chosen to give the same curvature at the well bottom of the Lippincott-Schroeder model potential: $D=110.6 \mathrm{kcal} / \mathrm{mol}$ and $\alpha=2.247 \AA^{-1}, 36,53,54$ yielding the frequency at the well bottom $\omega_{0}=3617 \mathrm{~cm}^{-1}$, and the lowest two frequencies $\omega_{10}=3447 \mathrm{~cm}^{-1}$ and $\omega_{21}=$ $3278 \mathrm{~cm}^{-1}$.

To solve the GLE with and without the GWP method the velocity-Verlet algorithm was used. The Runge-Kutta algorithm was used to solve the QME. The time step was $0.1 \mathrm{fs}$ in all simulations. The Gaussian white noise was generated for $r(t)$ in Eq. (18) and, therefore, the cumulant expansion in Eq. (9) is expected to be accurate. To calculate IR spectra, the volume $V$ in Eq. (1) was set to $15.0 \AA^{3}$, which is on the order of the molecular volume of water. In the TCF approach, the equilibrium simulation was carried out for 10000 ensembles of 10 ps trajectories. The TCFs were calculated with a time step of 0.5 fs. In the RF approach, the nonequilibrium simulation was conducted. Here, in the calculation of the time-dependent dipole moment $\langle M(t)-M(0)\rangle=$ $\langle\delta M(t)\rangle=\int_{-\infty}^{t} d t^{\prime} S\left(t-t^{\prime}\right) E\left(t^{\prime}\right)$, the electric field was approximated as the impulsive limit: $\langle\delta M(t)\rangle=\Delta t S(t) E$ with $E=0.2$ a.u. (for weak excitation) or 1 a.u. (for strong excitation in Secs. V C, V D, and V E) and $\Delta t=0.1$ fs. The electric field was equally applied in positive and negative directions along the stretching coordinate $Q$, which generates the responses $S_{+}$and $S_{-}$by Eq. (6), respectively. Then, the overall response was calculated to be $S_{+}-S_{-}$to counteract the even-order responses which do not change the sign regardless of the direction of the electric field. The number of ensembles was $5 \times 10^{6}$ for each direction. The nonequilibrium simulations were initiated with the equilibrium configurations sampled at the interval of $10 \mathrm{ps}$. The VER time was calculated by fitting the expectation value of Hamiltonian (total energy) to a single-exponential function for the GLE and by the memory time defined by $\int_{0}^{\infty} d t\langle H(t)\rangle /\langle H(0)\rangle$ for the QME. The total energy is defined as $P^{2} /(2 \mu)+V(Q)$ for the GLE, $\left(P^{2}+\pi^{2}\right) /(2 \mu)+\hbar^{2} /\left(8 \mu \rho^{2}\right)+V(Q, \rho)$ for the GLE with the GWP method, and $\langle H\rangle=\operatorname{Tr}[\rho H]$ for the QME. Similarly, in the simulation by the QME, the electric field is applied by adding the perturbation term to the Hamiltonian at the first time step. Then, the expectation value of coordinate $\operatorname{Tr}[\rho(t) Q]$ and the RF were calculated. The number of energy levels in the QME was set to 4 to which the convergence was achieved.

In calculating the frequency shift from the equilibrium trajectory, the matrix elements in Eqs. (7)-(9) were obtained by numerically solving the Schrödinger equation using the discrete variable representation ${ }^{55}$ for the Morse potential (see also Sec. V A). In the SFM approach, the VER time $T_{1}$ is necessary as an input, for which we adopt the value from the Landau-Teller formula in Eq. (16).

\section{RESULTS}

\section{A. Frequency time correlation function}

Before addressing the FTCF, we first check the accuracy of the perturbation theory in Eq. (8) by directly calculating the frequency shift by the GLE. ${ }^{17,18}$ Here, we use the adiabatic (Born-Oppenheimer) approximation as in the assumptions of the SFM approach described in Sec. II C. At each time step in the equilibrium simulation, the potential along the system coordinate $Q$ is computed while keeping the auxiliary momentum at its instantaneous value. The potential in our case is directly given by $V(Q)-Q \sqrt{\zeta \gamma} s$ from Eq. (17). The resulting vibrational Schrödinger equation is then solved numerically using the discrete variable representation. ${ }^{55}$ The results are shown in Fig. S2 (Ref. 51) and in good agreement between the instantaneous frequency shifts by the adiabatic approximation and the perturbation theory in Eq. (8). The maximum and average of the absolute value of error were 9.31 and $0.828 \mathrm{~cm}^{-1}$, respectively. The high-frequency oscillation can be seen in Fig. S2, which reflects the oscillation of the system via the system-bath coupling.

We now compute the FTCF from the instantaneous frequency shift and other sets of parameters. To investigate the relation between the bath parameters and the FTCF, we fit the resulting FTCF by the single-exponential function

$$
C(t)=\Delta^{2} \exp (-t / \tau)
$$

It is characterized by the root mean square (RMS) frequency fluctuation $\Delta$ and the correlation time $\tau$. Their dimensionless product $\tau \Delta$ determines whether the line shape is in either of the homogeneous $(\tau \Delta \gg 1)$ or inhomogeneous $(\tau \Delta \ll 1)$ limits. The single-exponential function is a good approximation for our system-bath model because the bath contains only one effective mode and the Gaussian white noise $r(t)$ was generated in Eq. (18). In contrast, another functional form is sometimes appropriate for the FTCF of molecular liquids because several kinds of (sometimes non-Gaussian) bath modes are involved. In our model spectral density in Eq. (15), $\zeta$ and $\gamma$ play a similar role as $\Delta$ and $1 / \tau$, respectively.

We note that the fast oscillation of the system oscillator appear in the FTCFs as in the trajectory of the instantaneous frequency shift. This kind of fast oscillation is usually not observed in the FTCF from MD simulations. The difference could arise from the following mechanism: The real bath oscillators have wider range of coupling modes, e.g., translation, libration, and other intramolecular vibrations, which are, however, treated as one effective mode in our model. Nevertheless, the overall decay of the FTCFs is dominated by the slow (low frequency) component and, therefore, the obtained $\Delta$ and $1 / \tau$ characterize the system-bath coupling.

The FTCFs for $\zeta / \gamma=0.5\left(\tilde{\gamma}=\gamma / \omega_{0}\right), 80(\tilde{\gamma}=0.05)$, $400(\tilde{\gamma}=0.008)$ by the GLE are shown in Fig. 1 . The reason of these choices of $\zeta / \gamma$ and $\tilde{\gamma}$ will become clear later in this section where comparison with previous studies in aqueous solutions is made. Those calculated with the GWP method 

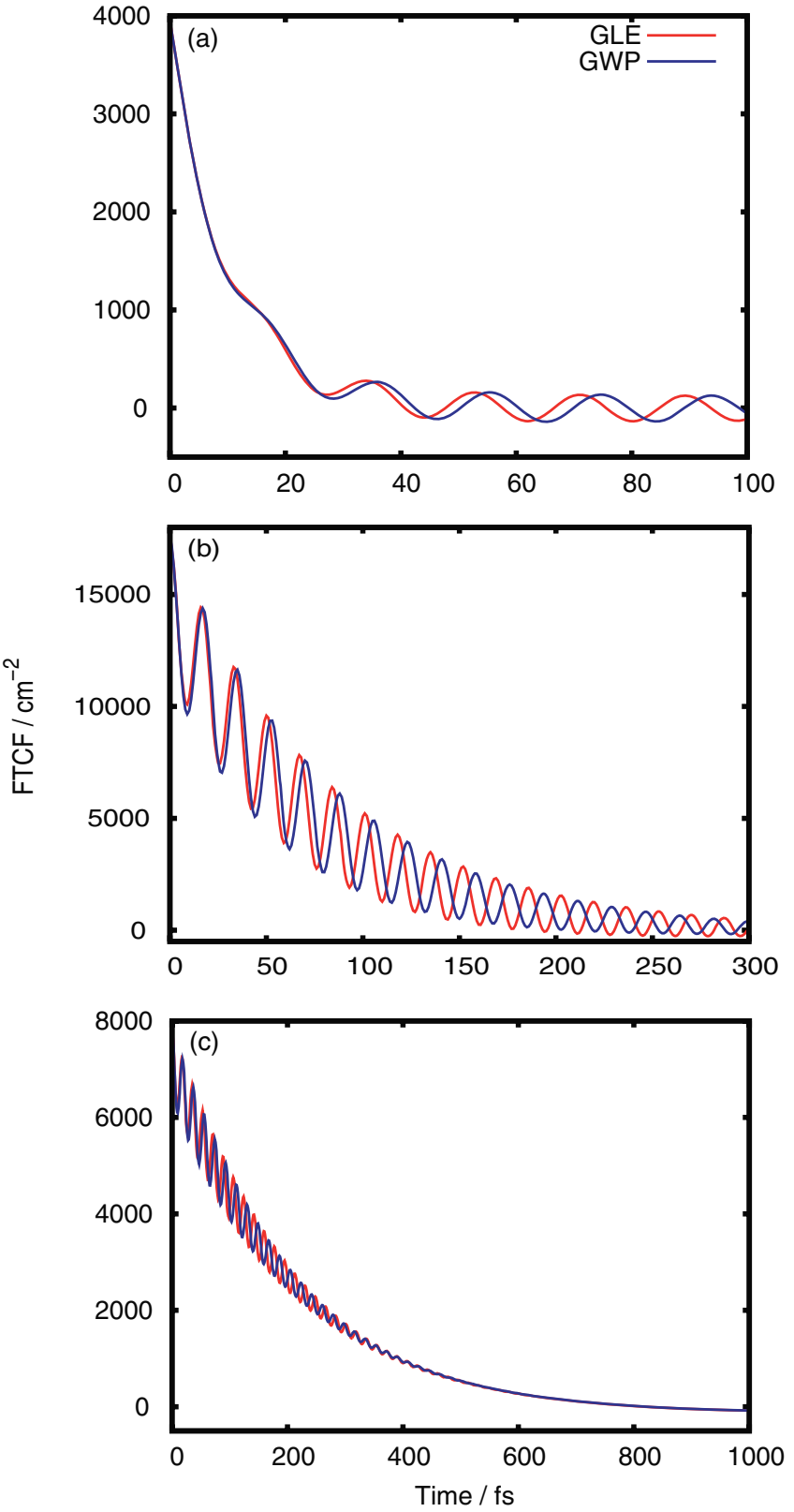

FIG. 1. Frequency time correlation functions (FTCFs) for (a) $\zeta / \gamma=0.5$ $\left(\tilde{\gamma}=\gamma / \omega_{0}\right),(\mathrm{b}) \zeta / \gamma=80(\tilde{\gamma}=0.05),(\mathrm{c}) \zeta / \gamma=400(\tilde{\gamma}=0.008)$.

are also shown in Fig. 1. The FTCFs with the GWP method and the classical ones agree well at short times and the overall decays are always quite similar. Only the frequency of the fast oscillation with the GWP method is different from that by the classical GLE, which becomes clearer at long times. This difference comes from inclusion of the zero-point effect in the former, which is also seen in the difference in the IR peak position in Figs. 3 and 5.

These FTCFs are fitted by Eq. (31) and the resulting parameters $\Delta$ and $\tau$ plotted as a function of $\tilde{\gamma}$ on a logarithmic scale in Fig. 2. The width parameter $\Delta$ increases as $\tilde{\gamma}$ increases and strongly depends on $\zeta / \gamma$. Conversely, $\tau$ decreases as a function of $\tilde{\gamma}$. The results in Fig. 2 and Table I indicate that $\zeta / \gamma$ is the major factor that determines whether the line shape is homogeneous or inhomogeneous, which is known to be determined by the product $\tau \Delta$. Indeed, we see in

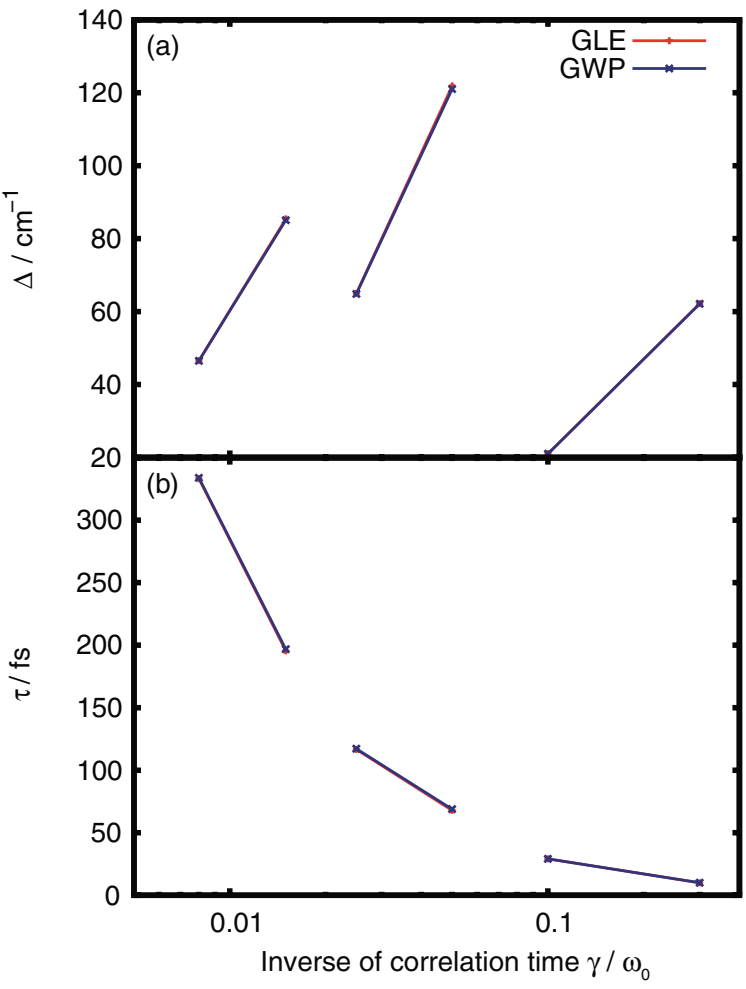

FIG. 2. Fitted bath parameters $\Delta$ and $\tau$ in Eq. (31) for the frequency mapping as a function of $\tilde{\gamma}$ on a logarithmic scale. The parameters are summarized in Table I. Note the difference in the values of $\zeta / \gamma$ : the points for the same $\zeta / \gamma$ are connected by lines.

Table I that $\tau \Delta$ depends mostly on $\zeta / \gamma$, which implies that they play a similar role. Furthermore, $1 / \tau$ plays a similar role as $\tilde{\gamma}$. From these considerations, we choose $\zeta / \gamma$ and $\tilde{\gamma}$ as variable parameters. The purpose of changing the bath parameters in this paper is to elucidate a general (and qualitative) trend with variation of the bath conditions, such as temperature, pressure, density, and polarity, which will be reflected in the resulting correlation time $\tau$, frequency fluctuation $\Delta$, IR spectra, and VER.

From the values of $\tau \Delta$ summarized in Table I, we categorize the bath parameters into the following three types:

- Homogeneous $(\tau \Delta \sim 0.1): \zeta / \gamma=0.5, \tilde{\gamma}=0.1$ or 0.3 .

- Intermediate $(\tau \Delta \sim 1): \zeta / \gamma=80, \tilde{\gamma}=0.025$ or 0.05 .

- Inhomogeneous $(\tau \Delta \sim 3): \zeta / \gamma=400, \tilde{\gamma}=0.008$ or 0.01 .

The typical examples of aqueous solutions which belong to the three types are $\mathrm{CN}^{-} / \mathrm{H}_{2} \mathrm{O}$ (homogeneous), $\mathrm{HOD} / \mathrm{D}_{2} \mathrm{O}$ (intermediate), and $\mathrm{OD}^{-} / \mathrm{HOD} / \mathrm{D}_{2} \mathrm{O}$ (inhomogeneous), respectively. In $\mathrm{CN}^{-} / \mathrm{H}_{2} \mathrm{O}, \tau \Delta=0.19\left(\Delta=\left\langle(\Delta \omega)^{2}\right\rangle^{1 / 2} \sim\right.$ $\left.15 \mathrm{~cm}^{-1}, \tau=43 \mathrm{fs}\right),{ }^{17}$ although another experimental group reported the different values closer to the second type by the double-exponential fitting: $\tau \Delta=0.4,2.7(\Delta=11,2$, $5.3 \mathrm{~cm}^{-1}$ and $\tau=0.2,2.9 \mathrm{ps}$, respectively). ${ }^{22}$ The fast component of the FTCF decay in $\mathrm{HOD} / \mathrm{D}_{2} \mathrm{O}$ gives $\tau \Delta \sim 1$ $\left(\Delta=141 \mathrm{~cm}^{-1}\right.$ and $\left.\tau=40 \mathrm{fs}\right){ }^{10,23} \mathrm{In} \mathrm{OD}^{-} / \mathrm{HOD} / \mathrm{D}_{2} \mathrm{O}$, $\tau \Delta \sim 2.5\left(\Delta \sim 65 \mathrm{~cm}^{-1}, \tau \sim 200 \mathrm{fs}\right) .^{9}$ 
TABLE I. Bath parameters and fitted parameters of the FTCFs in Eq. (31). $\tau \Delta$, which determines the line shape. The pure dephasing width $1 / T_{2}^{*}$ in Eq. (32) are also listed. $1 / T_{2}^{*}$ is in $\mathrm{cm}^{-1}$.

\begin{tabular}{lcccc}
\hline \hline$\tilde{\gamma}$ & $\zeta / \gamma$ & Line shape & $\tau \Delta$ & $1 / T_{2}^{*}$ \\
\hline 0.1 & 0.5 & homogeneous & 0.115 & 2.421 \\
0.3 & 0.5 & homogeneous & 0.117 & 7.280 \\
0.025 & 80 & intermediate & 1.43 & 93.11 \\
0.05 & 80 & intermediate & 1.63 & 196.4 \\
0.008 & 400 & inhomogeneous & 2.92 & 136.1 \\
0.01 & 400 & inhomogeneous & 3.20 & 273.3 \\
\hline \hline
\end{tabular}

For additional information, the pure (effective homogeneous) dephasing width ${ }^{22}$

$$
\frac{1}{T_{2}^{*}}=\Delta^{2} \tau
$$

are listed in Table I. This will be also discussed later in Fig. 8.

\section{B. Infrared spectra}

The IR spectra for (a) $\zeta / \gamma=0.5, \tilde{\gamma}=0.3$, (b) $\zeta / \gamma$ $=80, \tilde{\gamma}=0.05$, and (c) $\zeta / \gamma=400, \tilde{\gamma}=0.01$ are shown in Fig. 3. The position, amplitude, and full width at half maximum (FWHM) of the peaks in IR spectra as a function of $\tilde{\gamma}$ on a logarithmic scale are shown in Figs. 4(a), 4(b), and 4(c), respectively. The peak position and amplitude are measured at which the spectrum has its maximum. The results from the TCF approach were used for the GLE and GWP in Fig. 4 because the convergence of the spectra was better and the spectra were very close to the RF approach (see Fig. 3). The similarity between the two approaches indicates that the applied electric pulse is weak enough, that the system oscillator is perturbed weakly, and the linear response theory holds. However, they are not exactly the same: the TCF intensity is smaller in the higher frequency tail whereas the RF intensity is smaller in the lower frequency tail. This presumably reflects different characteristics of the two approaches in describing short and long time behaviors; for instance, the RF approach incorporates the short-time transient dynamics.

The dependencies of the peak position on $\tilde{\gamma}$ are very similar among all methods. On the other hand, the different dependencies of the amplitude and width on $\tilde{\gamma}$ are seen in Fig. 4. The IR spectra are further analyzed in terms of position, amplitude (height), and width (line shape) of peaks.

\section{Peak position}

As the coupling to the bath becomes strong ( $\tilde{\gamma}$ increases for a fixed $\zeta / \gamma)$, the peak position increases. The increase of peak position is due to the renormalization term in Eq. (11), which always gives a blue shift of frequency ${ }^{16}$ (see the discussion below). The difference between the positions calculated by the GLE and the QME ranges from 100 to $160 \mathrm{~cm}^{-1}$ and depends on the bath parameters. The GWP method gives

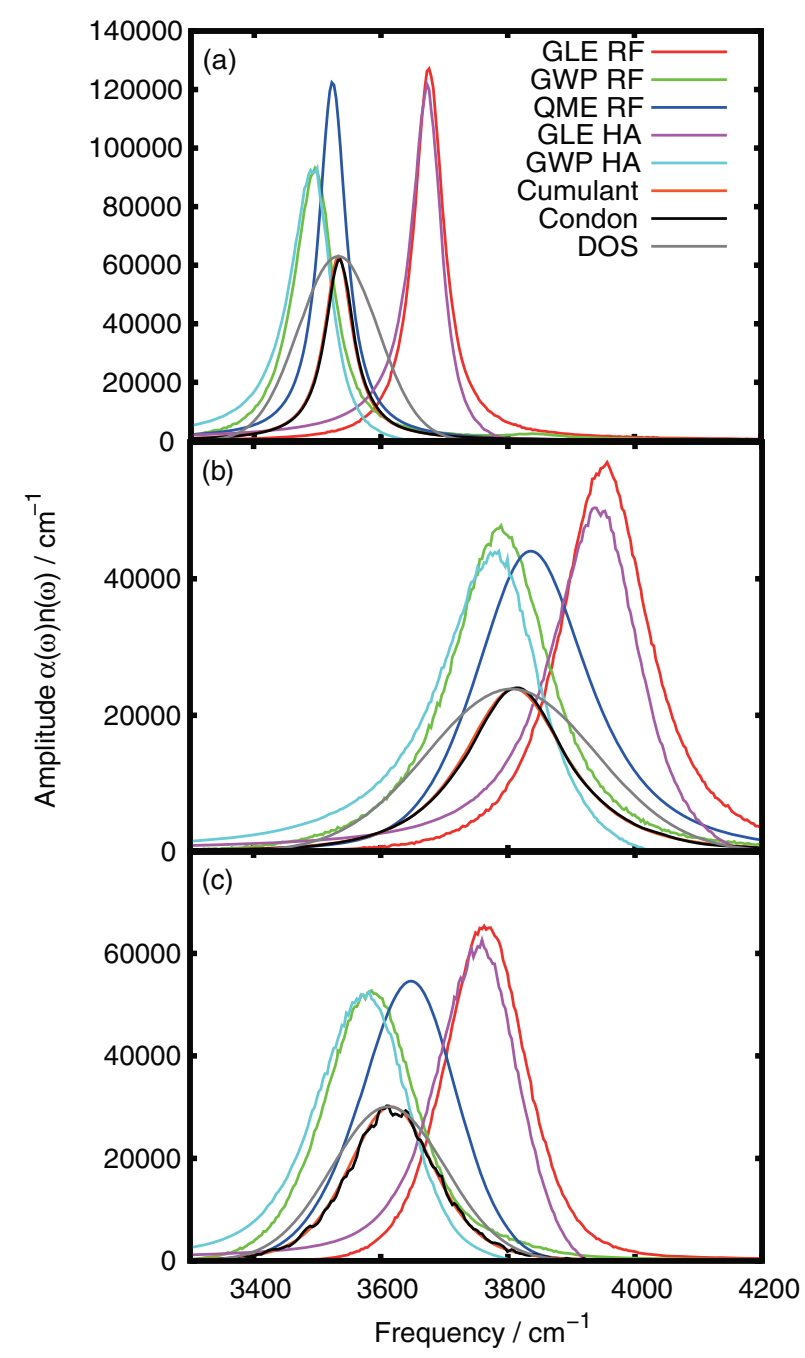

FIG. 3. IR spectra for (a) $\zeta / \gamma=0.5, \tilde{\gamma}=0.3$, (b) $\zeta / \gamma=80, \tilde{\gamma}=0.05$, and (c) $\zeta / \gamma=400, \tilde{\gamma}=0.01$. RF in the legend denotes the response function approach. HA represents harmonic approximation to the quantum correction factor in the TCF approach. Condon and cumulant denote that the spectra were calculated by Eqs. (7) and (9), respectively. Also shown is the densityof-states histogram for the frequency shift.

significant red shift from the classical GLE, yielding notable improvement but slight overshooting toward the QME reference. The red shift comes from the inclusion of zero-point effect and anharmonicity. The peak position for the SFM approach is not the computational outcome but an input parameter: it was set here to $\left\{\omega_{01}^{2}+Z(0) / \mu\right\}^{1 / 2}$ which can be derived from the Hamiltonian in Eq. (11). ${ }^{16}$ (In contrast, most of the previous studies on molecular systems have employed the peak position calculated by $a b$ initio electronic structure methods. ${ }^{23}$ )

The blue shift along the bath coupling strength stems from the term that renormalizes the Morse potential. This was needed to maintain the translational invariance of the theory, ${ }^{56}$ but at the cost of losing correspondence with the general experimental trend of red shift along the solvent polarity. Indeed, a previous study by Georgievskii et al. ${ }^{57}$ revealed that, without the renormalization term, the frequency of a cubic vibrational mode exhibits a blue or red shift depending on the system and bath parameters. On the other hand, with 


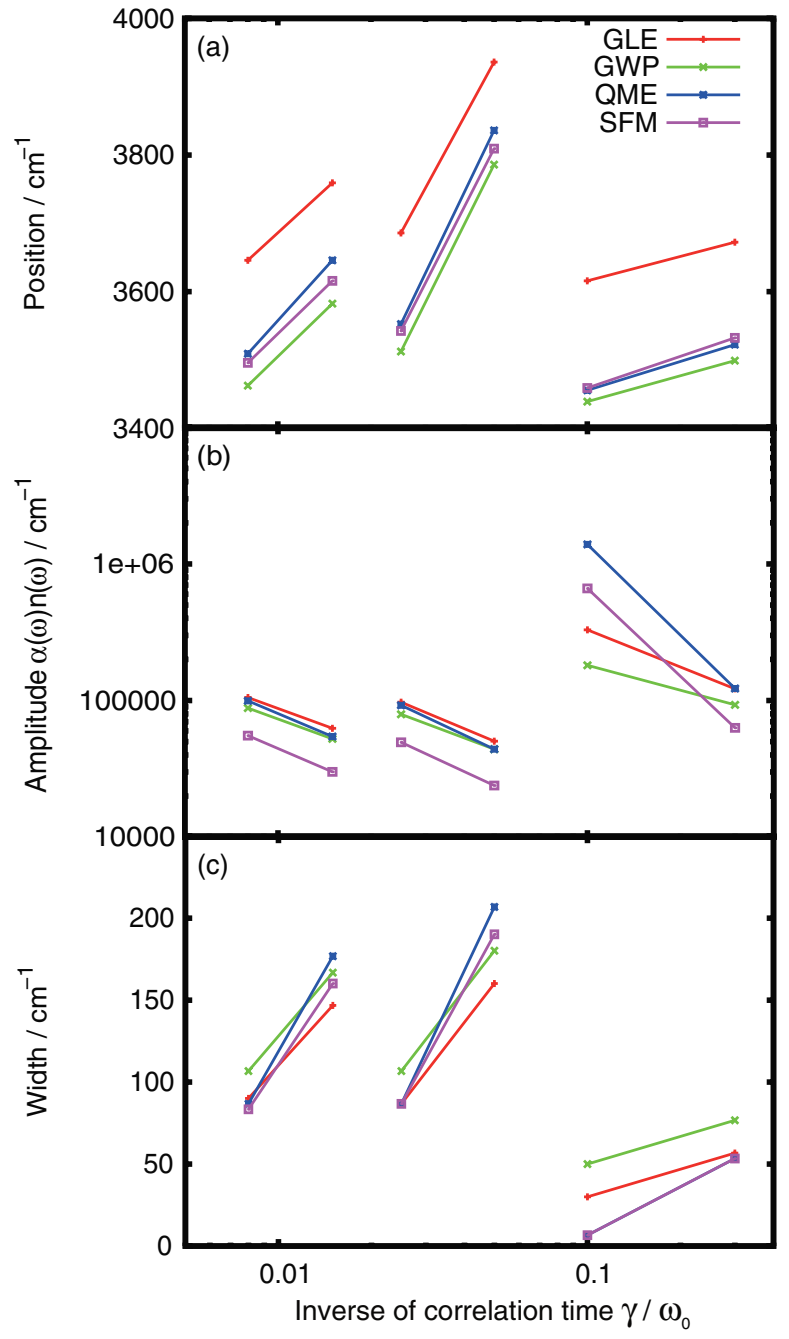

FIG. 4. IR (a) peak position, (b) amplitude, and (c) full width at half maximum as a function of $\tilde{\gamma}$ on a logarithmic scale. Note the peak amplitude in (b) is plotted on a logarithmic scale.

the renormalization term, the frequency was demonstrated to yield blue shift with the increasing system-bath coupling, in agreement with our results and others. ${ }^{58}$

\section{Peak amplitude}

As the coupling to the bath becomes strong, the IR amplitude decreases due to the increasing width. The IR amplitudes calculated by the TCF with the harmonic QCF agree well with those by the QME except the case with $\tilde{\gamma}=0.1$ and $\zeta / \gamma$ $=0.5$. This case represents large mismatch between the bath spectral peak (at $\omega=\gamma$ in $J(\omega)$ ) and the system frequency $\omega_{0}$ and weak system-bath coupling. It thus corresponds to the case of nonpolar liquids in which the longest lifetime of coherence is anticipated. Therefore, for the strong system-bath coupling as encountered in aqueous solutions, the harmonic QCF is expected to be accurate. For example, at the fundamental transition frequency $\omega_{10}=3447 \mathrm{~cm}^{-1}$ of our model system in the gas phase, $\beta \hbar \omega_{10}=16.53$, which indicates the QCF can be significant. In this case, the standard, harmonic, and Schofield QCFs yield $2 /\left\{1+\exp \left(-\beta \hbar \omega_{10}\right)\right\}=2.000$, $\beta \hbar \omega_{10} /\left\{1-\exp \left(-\beta \hbar \omega_{10}\right)\right\}=16.53, \quad$ and $\quad \exp \left(\beta \hbar \omega_{10} / 2\right)$ $=3887$, respectively. Hence, the harmonic QCF is the most accurate by orders of magnitude for the model. The adequacy of the harmonic QCF also carries over for the GWP method. Indeed, the harmonic QCF was previously used for the simulation of liquid water with the GWP method. ${ }^{19}$ The amplitude by the SFM is smaller than that by the QME by a factor of 1.7-2.1. This would arise from the treatment of the population and coherence relaxations as described in Sec. II C. Except this difference which is almost constant, the SFM can qualitatively reproduce the trends in the QME results.

\section{Peak width}

As the coupling to the bath becomes strong, the peak width increases. For large $\tilde{\gamma}$, the widths by the GLE are slightly larger than those by the QME. Conversely, for small $\tilde{\gamma}$, the formers are slightly smaller than the latters. The GWP method always broadens the IR spectra compared to the classical GLE. This is mainly due to the faster dephasing coming from the better inclusion of the anharmonicity as was also noted in the discussion of the peak position. The widths by the SFM are the closest to those by the QME both in number and dependence on $\tilde{\gamma}$.

\section{Anharmonicity}

For a sufficiently weak pulse, the 1-2 transition is negligible because the population of the excited state is quite small. On the other hand, if the system is excited by a strong impulsive pulse, the 1-2 transition cannot be ignored. Also when anharmonicity is quite strong, the higher excited states of vibration becomes important mainly due to the nonadiabatic transition between the vibrational states. This is relevant, for instance, to the IR-induced proton transfer, in which after the excitation the proton transfer occurs and the proton potential becomes quite anharmonic. ${ }^{3-5,59}$ Therefore, we investigate the vibrational dynamics induced by a strong pulse. The IR spectra by a strong pulse excitation are shown in Fig. 5. The results from the TCF method (same as in Fig. 3) are also shown for comparison.

The convergence of the IR spectra is better than by a weak pulse because the response is larger. The peak positions calculated by the RF are slightly red-shifted compared to those by the TCF. In addition, the line shapes are more distorted towards the low-frequency side. This is because the RF approach includes the nonlinear interactions caused by the anharmonicity along the vibrational coordinate, especially when the vibrational coordinate is stretched largely. The peak at $\omega_{12}$ ( $\sim 3400 \mathrm{~cm}^{-1}$ for Fig. 5(a)) appears only by the QME. This suggests that the 1-2 transition can only be reproduced by the quantum mechanical treatment of the system oscillator. ${ }^{60}$ On the other hand, the spectrum by the GWP is significantly broadened and have notable amplitude in the region corresponding to $\omega_{12}$, but fails to show the separate peaks structure. This can possibly be remedied via inclusion of the quantum phase in the semiquantal formalism. ${ }^{61}$ As expected and seen 


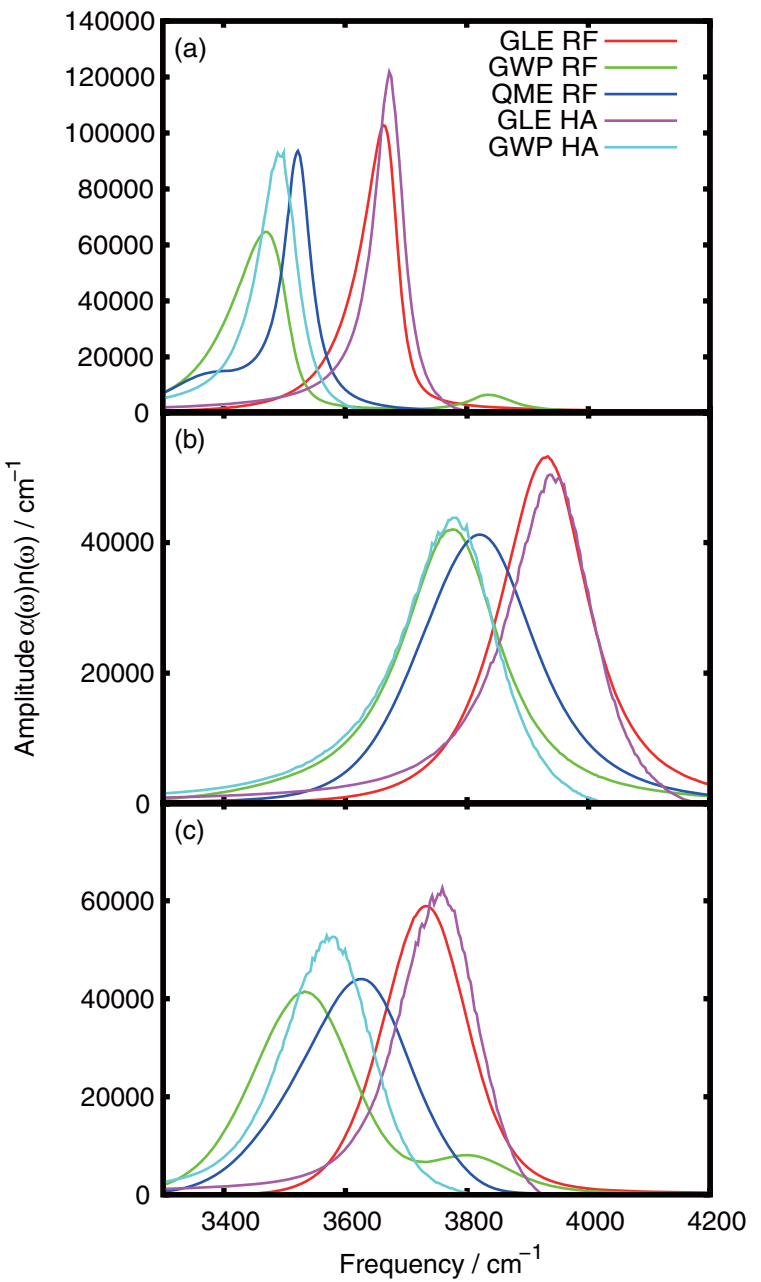

FIG. 5. IR spectra for the same parameters in Fig. 3. In the RF approach, the strong pulse was used.

in the figure, the 1-2 transition is out of the applicability of the classical GLE approach.

\section{Extra peak in the Gaussian wavepacket method}

The IR spectra calculated by the GWP method exhibit a small extra peak at the higher frequency than that of the fundamental transition. Especially, the peak manifests itself when the system oscillator is excited by a strong pulse. Therefore, we show the blowup of the extra peaks, which are calculated by the RF approach with the GWP method in Fig. 6(a). Here, for $\tilde{\gamma}=0.05$ (corresponding to Fig. 3(b)) the extra peak merges with the peak of the fundamental transition centered at $3779 \mathrm{~cm}^{-1}$. These peaks are reminiscent of the previously observed in liquid water. ${ }^{19}$ In liquid water, the higher-frequency peak around $4400 \mathrm{~cm}^{-1}$ was attributed to the energy exchange between the wavepacket width dynamics and the coupling of the symmetric $\mathrm{OH}$ stretching mode at $3596 \mathrm{~cm}^{-1}$ and the rotational motion at $808 \mathrm{~cm}^{-1}$.

The positions of the peaks in Fig. 6(a) are almost independent of $\tilde{\gamma}$, which ranges from 0.08 to 0.3 . Thus, the peaks cannot be attributed to the low-frequency bath mode. Instead, it arises from the difference frequency between the motions

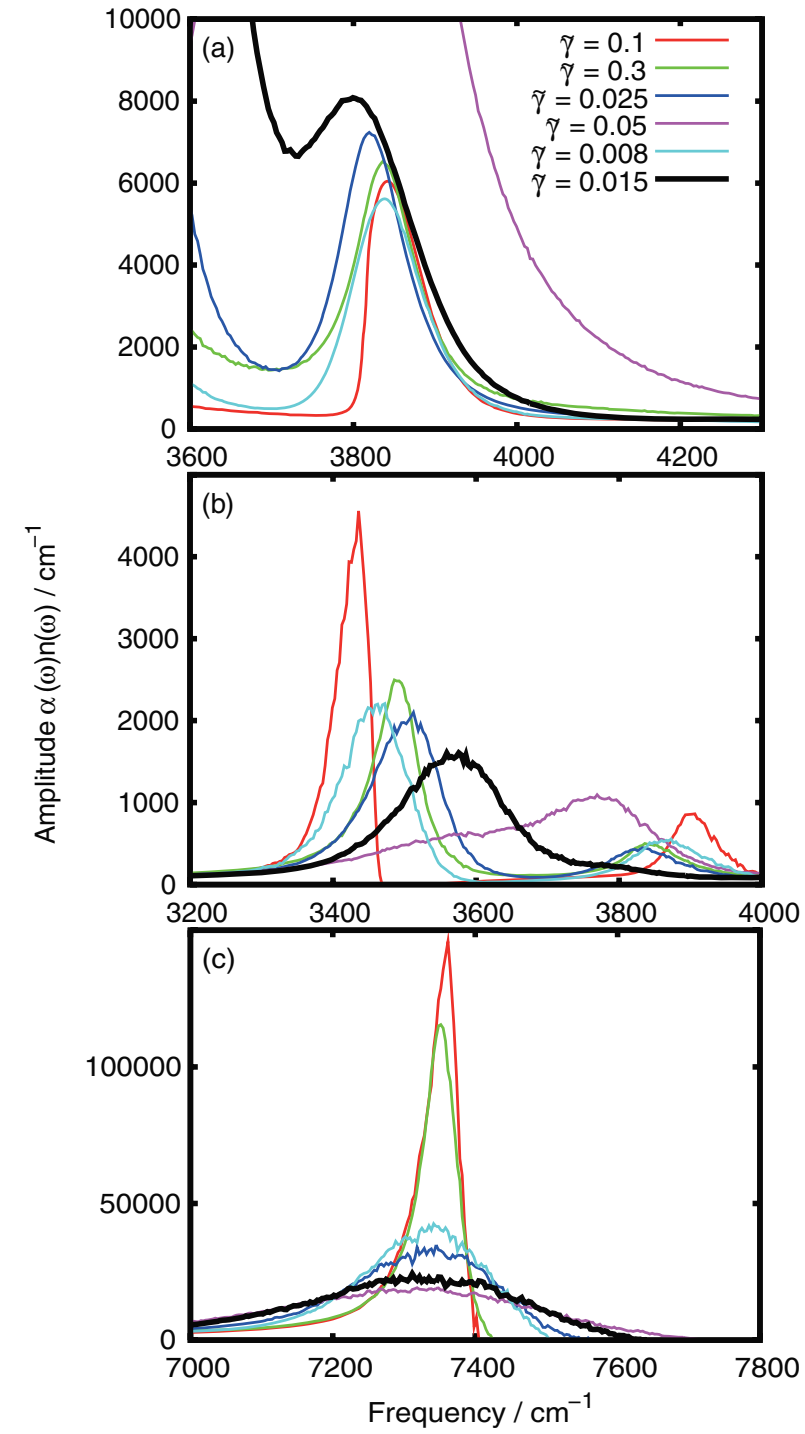

FIG. 6. (a) Blowup of the extra peaks excited by the strong pulse in Fig. 5. These spectra are calculated by the RF approach with the GWP method. Fourier transforms of the wavepacket width TCFs in the frequency ranges (b) $3200-4000 \mathrm{~cm}^{-1}$ and (c) $7000-7800 \mathrm{~cm}^{-1}$.

of the center $Q$ and width $\rho$ coordinates. The (quenched) normal mode analysis ${ }^{43}$ on $V(Q, \rho)$ yields the frequencies of 3642 and $7668 \mathrm{~cm}^{-1}$ whose difference is $4026 \mathrm{~cm}^{-1}$. Taking account of the blue shift of $\omega_{01}$ from the gas-phase value $3447 \mathrm{~cm}^{-1}$ to $3583 \mathrm{~cm}^{-1}$ when $\tilde{\gamma}=0.015$, the difference frequency may red shift to $3890 \mathrm{~cm}^{-1}$ given that the frequency of the width remains the same. The difference corresponds to the observed higher-frequency peaks in Fig. 6(a) ranging from 3800 to $3878 \mathrm{~cm}^{-1}$.

To confirm the anharmonic shift of frequency from that calculated by the normal mode analysis, the Fourier transform of the wavepacket width TCF is shown in Figs. 6(b), and 6(c) with different frequency ranges. These spectra were calculated by simply replacing the dipole TCF in Eq. (1) by the wavepacket width TCF. The peak positions in Fig. 6(b) are similar to those in Figs. 5 and 6(a). In contrast, the positions of the extra peaks do not depend much on the bath parameters and range from 7330 to $7360 \mathrm{~cm}^{-1}$. This 


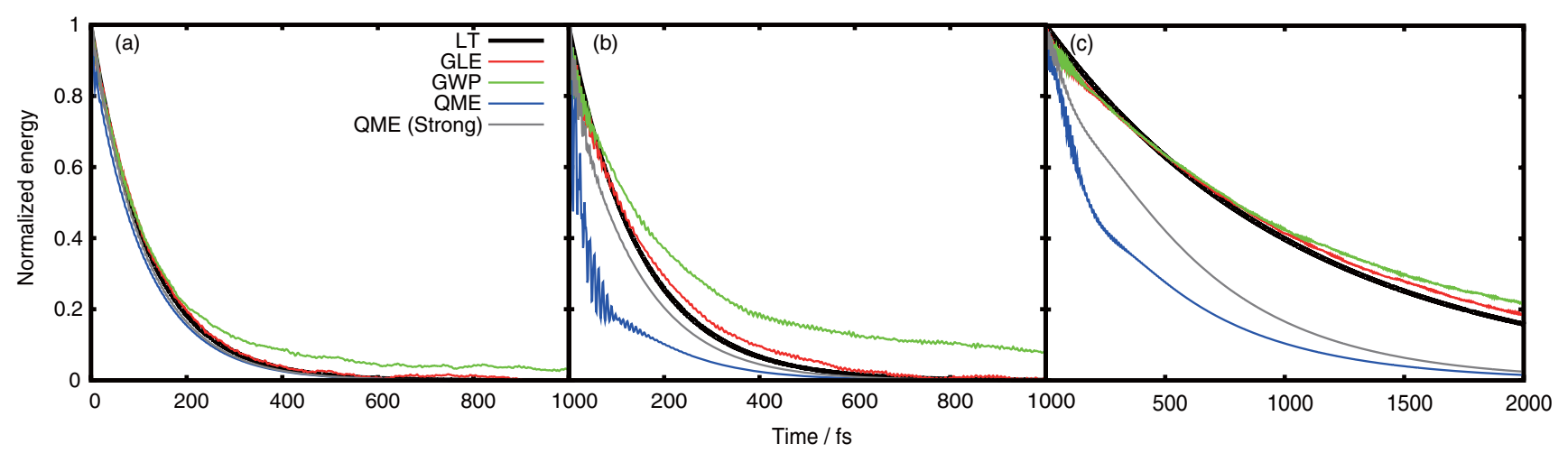

FIG. 7. Time evolution of normalized energy after excitation for the same parameters in Fig. 3. The results calculated by the Landau-Teller formula in Eq. (16) and the QME with a strong excitation pulse are also shown.

independence is because the bath is only attached to the center of the wavepacket as seen in Eq. (26). Moreover, a small shoulder at approximately $3600 \mathrm{~cm}^{-1}$ in Fig. 6(b) for $\tilde{\gamma}=0.05$ can be seen, which corresponds to the extra peak because the peak of the fundamental transition is blue-shifted to approximately $3800 \mathrm{~cm}^{-1}$. This confirms that the extra peaks appear at the difference frequency of the two modes in the space of the center and width of a wavepacket.

Unfortunately, the approximations involved implies that the present QME simulation is insufficient to describe the nonequilibrium wave packet breathing dynamics induced by the strong pulse excitation. This issue is thus left open to future investigations.

\section{E. Motional narrowing from the semiclassical frequency modulation model}

The coincidence of the IR spectra by the cumulant approximation with those by the Condon approximation is due to the Gaussian property of an auxiliary momentum by our simulation method. Actually, in producing the white noise $r(t)$ in Eq. (18) we have generated the Gaussian noise. To reproduce experimental or calculated IR spectra, the nonCondon effect should be considered, which manifests itself in, for example, $\mathrm{HOD} / \mathrm{D}_{2} \mathrm{O} .{ }^{29}$ Although the amplitude is clearly underestimated, the correct behavior along the bath parameters observed in Fig. 4 would encourage the use of this method for studying dependence of IR spectra on external parameters such as pressure or isotope substitution.

To see the effect of the motional narrowing on IR spectra, we also show the density-of-states (DOS) histogram for the frequency shift in Fig. 3. This was calculated by the time series of the instantaneous frequency shift in the equilibrium simulation. The amplitude is normalized to be the same as that by the SFM. As is well known, the motional narrowing is the most significant in Fig. 3(a) by the homogeneous broadening. The degree of the motional narrowing can be estimated by the ratio of FWHM of the IR spectra to that of the DOS histogram: $\left(56.7 \mathrm{~cm}^{-1}\right) /\left(148.0 \mathrm{~cm}^{-1}\right)$ $=38.3 \%, \quad\left(196.8 \quad \mathrm{~cm}^{-1}\right) /\left(311.6 \quad \mathrm{~cm}^{-1}\right)=63.2 \%, \quad$ and $\left(160.1 \mathrm{~cm}^{-1}\right) /\left(209.6 \mathrm{~cm}^{-1}\right)=76.1 \%$ for Figs. 3(a), 3(b), and $3(\mathrm{c})$, respectively.
For example, the SFM approach was applied to $\mathrm{HOD} / \mathrm{D}_{2} \mathrm{O}$ and the motional narrowing affects the simulated line shape compared to the experimental spectra. ${ }^{18}$ In this case, the ratio was $\left(130 \mathrm{~cm}^{-1}\right) /\left(240 \mathrm{~cm}^{-1}\right)=54.2 \%$. If the $T_{1}$ relaxation is included, the FWHM would increase approximately by $1 /\left(2 T_{1}\right)=22.7 \mathrm{~cm}^{-1}$ given the experimental $T_{1}=750 \mathrm{fs}$ for the $\mathrm{OH}$ stretch of HOD in $\mathrm{D}_{2} \mathrm{O} .^{23,62}$ Then, the ratio would become $\left(152.7 \mathrm{~cm}^{-1}\right) /\left(262.7 \mathrm{~cm}^{-1}\right)=58.1 \%$. Considering that the IR line shape of the $\mathrm{OH}$ stretch of HOD in $\mathrm{D}_{2} \mathrm{O}$ is intermediate or inhomogeneous, ${ }^{52}$ this value is still smaller than the corresponding values in our calculations ( 63.2 or $76.1 \%$, respectively). One of the origins of this difference would be the simplicity of our model that tends to underestimate motional narrowing.

\section{F. Energy relaxation time}

The time evolution of normalized energy after excitation is shown in Fig. 7 for the same parameters in Fig. 3. The results calculated by the Landau-Teller formula in Eq. (16) and the QME with a strong excitation pulse are also shown. These results were fitted by a single-exponential function for the GLE and by the memory time defined by $\int_{0}^{\infty} d t\langle H(t)\rangle /\langle H(0)\rangle$ for the QME because the decay of energy contains a non-exponential decay. The resulting VER time $T_{1}$ as a function of $\tilde{\gamma}$ on a logarithmic scale is shown in Fig. 8(a). The dephasing time $T_{2}^{*}$ by Eq. (32) in Sec. V A is also shown for comparison. As $\tilde{\gamma}$ increases with $\zeta / \gamma$ fixed, $T_{1}$ decreases since the coupling to the bath becomes stronger. When $\tilde{\gamma}$ is large, all $T_{1}$ 's from the different methods agree well with each other. On the other hand, the LT, GLE, GWP, and QME results differ from each other when $\tilde{\gamma}$ is intermediate or small. One notable distinction is seen in the long time behavior in the GWP method; it slows down the VER since the energy is deposited in the width variable as seen in Eq. (26). This is because no bath oscillator is directly attached to the width variable, which is a particular feature of the bilinear coupling model as noted around Eq. (26). Further, $T_{1}$ 's calculated by the QME are different from others due to the non-Markovian effect. ${ }^{1,63}$ For example, if the energy relaxation curve is fitted for $\tilde{\gamma}=0.05$ by two exponential functions $w_{1} \exp \left(-t / t_{1}\right)+w_{2} \exp \left(-t / t_{2}\right)$ 


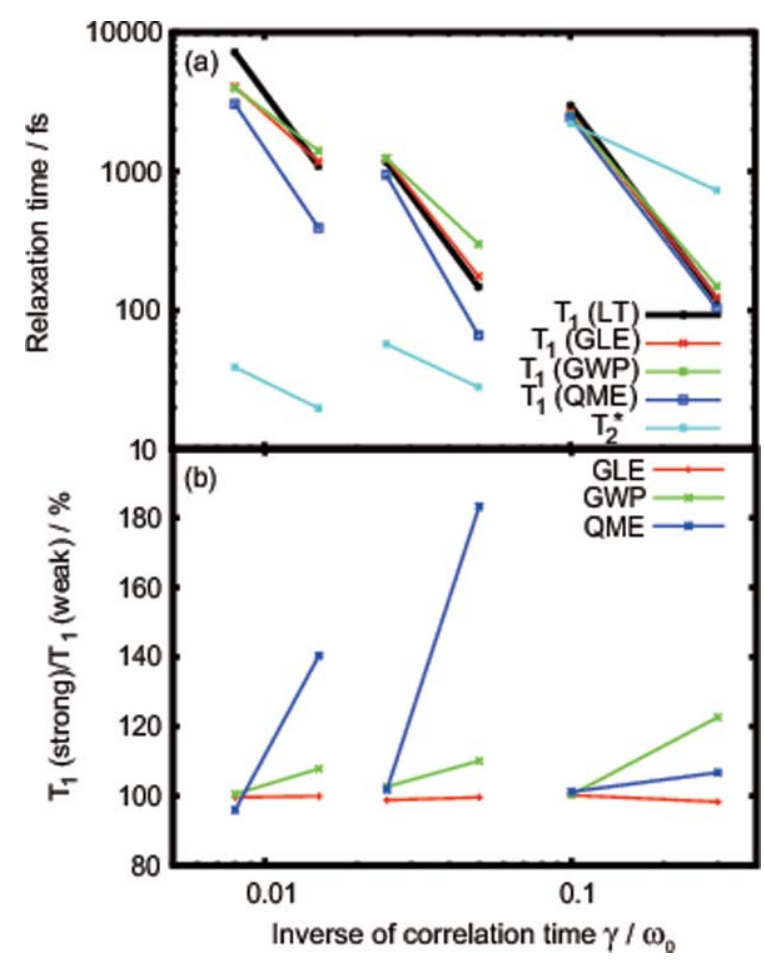

FIG. 8. (a) Energy relaxation time as a function of $\tilde{\gamma}$ on a logarithmic scale. Also shown is the dephasing time $T_{2}^{*}$ by Eq. (32) in Sec. V A for comparison. (b) Ratio of $T_{1}$ between by the weak pulse excitation and by the strong one in percentage.

where $w_{1}, w_{2}, t_{1}$, and $t_{2}$ are the fitting parameters, then we find $t_{1}, t_{2}=29.9,173.6$ fs. Because $R_{a b, c d}(t)$ in Eq. (28) approaches its Markovian limit for $t \gg 1 / \gamma$, the slower decay constant is more related to the Markovian value. Actually, the slower decay constant $t_{2}$ is comparable to that by the LT formula, $147.2 \mathrm{fs}$. It is noted that $T_{2}^{*}$ is smaller than $T_{1}$ for small $\tilde{\gamma}$ and comparable or larger than $T_{1}$ for large $\tilde{\gamma}$. Despite this, the SFM model was shown to work well for all the bath parameters.

One of the interesting problems in VR is the dependence of the VER rate on the strength of an excitation pulse. For instance, the VER rate of a Morse oscillator was reported to be an increasing function of the excitation energy for the Drude bath whereas it is a decreasing function for the Ohmic bath. ${ }^{32}$ This indicates that the non-Markovian effect by the colored bath plays an important role in VER. Hence, we examine the dependence of the VER rate on the pulse strength by the ratio of $T_{1}$ between the weak and strong pulse excitation cases. The result (in percentage) is shown in Fig. 8(b). Most of the results by the GLE are less than $100 \%$ especially for small $\tilde{\gamma}$ (the correlation time is long). The value for large $\tilde{\gamma}$ is close to $100 \%$ because the non-Markovian effect is less than that for small $\tilde{\gamma}$. The results by the GWP method are usually larger than $100 \%$. This is because as the excitation energy becomes large the energy deposited in the width variable increases and the VER rate slows down. The results by the QME for $\tilde{\gamma}$ $=0.015$ and 0.05 are much larger than $100 \%$ because the short-time decays are reduced as seen in Figs. 7(b) and 7(c).

Although the quantum and classical $T_{1} \mathrm{~s}$ are identical for a harmonic oscillator, ${ }^{27}$ the derivation assumed the Markov approximation that the bath relaxes much faster than the time scale of transitions between quantum states of the system oscillator. When the line shape is inhomogeneous $(\tilde{\gamma}=0.3)$, the ratio $\left(T_{1}^{\mathrm{GLE}} / T_{1}^{\mathrm{QME}}\right)$ of $T_{1}$ calculated by the $\mathrm{GLE}\left(T_{1}^{\mathrm{GLE}}\right)$ to that by the $\mathrm{QME}\left(T_{1}^{\mathrm{QME}}\right)$ is 1.19 by the weak pulse excitation in Fig. 7(a). Otherwise, the ratios are much greater than 1 (2.66 and 3.00 for $\tilde{\gamma}=0.05$ and 0.008 in Figs. 7(b) and 7(c), respectively). This suggests that the quantum effect for $T_{1}$ is larger when the line shape is inhomogeneous than when it is homogeneous. In the Appendix, we also compare the energy of our pulse excitation with that by Bader, Berne, Pollak, and Hänggi (BBPH) ${ }^{32}$ which employs the same model as ours in different conditions.

\section{CONCLUDING REMARKS}

In summary, we have investigated the vibrational spectroscopy and relaxation of an anharmonic oscillator in liquids by the GLE, the GWP method, and the QME. IR spectra have been calculated and analyzed by the TCF, the RF, and the SFM. Molecular vibrations have been modeled by a Morse oscillator coupled linearly to a harmonic oscillator bath. The FTCFs are calculated by the perturbation theory, which is shown to yield the accurate instantaneous frequency shift. Fitting the FTCF yields the RMS frequency fluctuation $\Delta$ and correlation time $\tau$ of the model. Then, we have categorized the bath parameters based on the criteria whether the line shape is homogeneous or inhomogeneous and calculated the pure dephasing width. The harmonic approximation to the QCF in the TCF approach is shown to work well to simulate IR spectra. The peak amplitude and width in the IR spectra calculated by the GLE are shown to agree well with those by the QME. However, the vibrational frequency is overestimated. It is found that the GWP method improves the peak position by taking account of the zero-point energy and the anharmonicity, yet the red-shift slightly overshoots the QME reference. Moreover, the GWP method yields an extra peak especially when a strong pulse is used, which arises from the difference frequency of the motions of the center and width of the wavepacket. The SFM approach is shown to underestimate the peak amplitude of IR spectra but reproduce the correct width of the peak and its dependence on the bath parameters. When the system is excited by a strong pulse, the peak in the lower frequency region due to the 1-2 transition appears only by the QME. The motional narrowing has been slightly underestimated compared to other simulations of molecular vibrations due to the simplicity of our model. Moreover, it is found that the strong pulse excitation accelerates the VER calculated by the GLE. A non-exponential decay at short times is found in the energy of the oscillator by the QME after excitation with a weak pulse when the correlation time of the bath is long.

For the simulation of IR spectra, using the QME as a reference, the GWP and SFM approaches yield a qualitatively correct fundamental transition frequency. The classical GLE predicts the higher frequency than other methods for an anharmonic oscillator studied. Conversely, for the simulation of the VER rate and its excitation energy dependence, the classical GLE gives a qualitatively reasonable description. 


\section{ACKNOWLEDGMENTS}

T.J. was supported by the Research Fellowship of the Japan Society for the Promotion of Science and the Grants in Aid for Scientific Research (KAKENHI) from the Ministry of Education. K.A. acknowledges supports from KAKENHI Nos. 20108017 (“ $\pi$-space”) and 22550012.

\section{APPENDIX A: EXCITATION ENERGY}

In this appendix, we compare our results with those of Bader, Berne, Pollak, and Hänggi. ${ }^{32}$ First, the Morse parameters are different from ours: $D^{\mathrm{BBPH}}=49.2 \mathrm{kcal} / \mathrm{mol}$ and $\omega_{0}^{\mathrm{BBPH}}=296 \mathrm{~cm}^{-1}$. Second, the excitation energy is far beyond ours: the ratios of the excitation energy to $\hbar \omega_{0}$ of BBPH was $10,40,80 k_{\mathrm{B}} T / \hbar \omega_{0}^{\mathrm{BBPH}}=7.0,28.2,56.4$. On the other hand, ours were $\left\{\Delta P^{2} /(2 \mu)\right\} / \hbar \omega_{0}=2.8 \times 10^{-3}, 7.1 \times 10^{-2}$. Here, we assumed that the excitation energy approximately given by the increased moment $\Delta P$ as $\Delta P \sim \Delta t e E / 2$. Our values are far less than those of BBPH. In addition, the excitation energy of BBPH is close to the dissociation limit of the oscillator and significantly large because experiments are usually conducted near equilibrium. Thus, our smaller excitation energy corresponds to experimental situations better. Therefore, our simulation methodology has shown the increase of the VER rate with increasing excitation energy in a more realistic situation.

${ }^{1}$ D. W. Oxtoby, Adv. Chem. Phys. 47, 487 (1981).

${ }^{2}$ J. L. Skinner and K. Park, J. Phys. Chem. B 105, 6716 (2001).

${ }^{3}$ K. Ando and J. T. Hynes, J. Phys. Chem. A 103, 10398 (1999).

${ }^{4}$ T. Joutsuka and K. Ando, J. Phys. Chem. A 115, 671 (2011).

${ }^{5}$ T. Joutsuka and K. Ando, J. Phys. Chem. A 115, 678 (2011).

${ }^{6}$ E. H. G. Backus, P. H. Nguyen, V. Botan, R. Pfister, A. Moretto, M. Crisma, C. Toniolo, G. Stock, and P. Hamm, J. Phys. Chem. B 112, 9091 (2008).

${ }^{7}$ A. G. Dijkstra, T. L. C. Jansen, and J. Knoester, J. Phys. Chem. A 114, 7315 (2010)

${ }^{8}$ M. Khalil, N. Demirdöven, and A. Tokmakoff, J. Phys. Chem. A 107, 5258 (2003)

${ }^{9}$ H.-K. Nienhuys, A. J. Lock, R. A. van Santen, and H. J. Bakker, J. Chem. Phys. 117, 8021 (2002).

${ }^{10}$ C. P. Lawrence and J. L. Skinner, Proc. Natl. Acad. Sci. U.S.A. 102, 6720 (2005)

${ }^{11}$ J. A. Poulsen, G. Nyman, and S. Nordholm, J. Phys. Chem. A 107, 8420 (2003)

${ }^{12} \mathrm{~S}$. Mukamel, Principles of Nonlinear Optical Spectroscopy (Oxford, New York, 1995).

${ }^{13}$ D. W. Oxtoby, D. Levesque, and J.-J. Weis, J. Chem. Phys. 68, 5528 (1978).

${ }^{14}$ D. W. Oxtoby, J. Chem. Phys. 70, 2605 (1979).

${ }^{15}$ D. W. Oxtoby, Adv. Chem. Phys. 40, 1 (1979).

${ }^{16}$ A. M. Levine, M. Shapiro, and E. Pollak, J. Chem. Phys. 88, 1959 (1988).

${ }^{17}$ R. Rey and J. T. Hynes, J. Chem. Phys. 108, 142 (1998).

${ }^{18}$ K. B. Møller, R. Rey, and J. T. Hynes, J. Phys. Chem. A 108, 1275 (2004).

${ }^{19}$ K. Hyeon-Deuk and K. Ando, J. Chem. Phys. 131, 064501 (2009).
${ }^{20}$ M. Tuckerman and B. J. Berne, J. Chem. Phys. 98, 7301 (1993).

${ }^{21}$ R. Kubo, Adv. Chem. Phys. 15, 101 (1969).

${ }^{22}$ M. Kozinski, S. Garrett-Roe, and P. Hamm, Chem. Phys. 341, 5 (2007).

${ }^{23}$ S. A. Corcelli, C. P. Lawrence, and J. L. Skinner, J. Chem. Phys. 120, 8107 (2004).

${ }^{24}$ A. Nitzan, Chemical Dynamics in Condensed Phases (Oxford University Press, New York, 2006).

${ }^{25}$ V. May and O. Kühn, Charge and Energy Transfer Dynamics in Molecular Systems, 2nd ed. (Wiley-VCH, Weinheim, 2004).

${ }^{26}$ R. Ramirez, T. Lopez-Ciudad, P. Kumar P, and D. Marx, J. Chem. Phys. 121, 3973 (2004).

${ }^{27}$ J. S. Bader and B. J. Berne, J. Chem. Phys. 100, 8359 (1994).

${ }^{28}$ S. A. Egorov, K. F. Everitt, and J. L. Skinner, J. Phys. Chem. A 103, 9494 (1999).

${ }^{29}$ J. R. Schmidt, S. A. Corcelli, and J. L. Skinner, J. Chem. Phys. 123, 044513 (2005).

${ }^{30}$ J. Skinner, Theor. Chem. Accounts 128, 147 (2011).

${ }^{31}$ E. L. Sibert, S. G. Ramesh, and T. S. Gulmen, J. Phys. Chem. A 112, 11291 (2008).

${ }^{32}$ J. S. Bader, B. J. Berne, E. Pollak, and P. Hänggi, J. Chem. Phys. 104, 1111 (1996).

${ }^{33}$ F. Marchesoni and P. Grigolini, J. Chem. Phys. 78, 6287 (1983).

${ }^{34}$ M. Ceriotti, G. Bussi, and M. Parrinello, Phys. Rev. Lett. 102, 020601 (2009).

${ }^{35}$ K. Ando, J. Chem. Phys. 121, 7136 (2004).

${ }^{36}$ K. Ando, J. Chem. Phys. 125, 014104 (2006).

${ }^{37}$ E. J. Heller, J. Chem. Phys. 62, 1544 (1975).

${ }^{38}$ E. J. Heller, J. Chem. Phys. 64, 63 (1976).

${ }^{39}$ I. Burghardt, H.-D. Meyer, and L. S. Cederbaum, J. Chem. Phys. 111, 2927 (1999)

${ }^{40}$ I. Burghardt, M. Nest, and G. A. Worth, J. Chem. Phys. 119, 5364 (2003).

${ }^{41}$ O. V. Prezhdo and Y. V. Pereverzev, J. Chem. Phys. 113, 6557 (2000).

${ }^{42}$ E. M. Heatwole and O. V. Prezhdo, J. Phys. Soc. Jpn. 77, 044001 (2008).

${ }^{43}$ Y. Shigeta, H. Miyachi, T. Matsui, and K. Hirao, Bull. Chem. Soc. Jpn. 81, 1230 (2008).

${ }^{44}$ Y. Shigeta, H. Miyachi, and K. Hirao, J. Chem. Phys. 125, 244102 (2006).

${ }^{45}$ D. Egorova, M. Thoss, W. Domcke, and H. Wang, J. Chem. Phys. 119, 2761 (2003).

${ }^{46}$ U. Kleinekathofer, J. Chem. Phys. 121, 2505 (2004).

${ }^{47}$ A. Ishizaki and Y. Tanimura, Chem. Phys. 347, 185 (2008).

${ }^{48}$ J. Liebers, U. Kleinekathöfer, and V. May, Chem. Phys. 347, 229 (2008).

${ }^{49}$ G. Nan, Q. Shi, and Z. Shuai, J. Chem. Phys. 130, 134106 (2009).

${ }^{50}$ M. Ban, S. Kitajima, and F. Shibata, Phys. Lett. A 374, 2324 (2010).

${ }^{51}$ See supplementary material at http://dx.doi.org/10.1063/1.3594093 for the time evolution of energy of a harmonic oscillator and the instantaneous frequency shift.

${ }^{52}$ C. P. Lawrence and J. L. Skinner, J. Chem. Phys. 117, 8847 (2002).

${ }^{53}$ E. R. Lippincott and R. Schroeder, J. Chem. Phys. 23, 1099 (1955).

${ }^{54}$ H. J. Bakker and H.-K. Nienhuys, Science 297, 587 (2002).

${ }^{55}$ D. T. Colbert and W. H. Miller, J. Chem. Phys. 96, 1982 (1992).

${ }^{56}$ C. Meier and D. J. Tannor, J. Chem. Phys. 111, 3365 (1999).

${ }^{57}$ Y. I. Georgievskii and A. A. Stuchebrukhov, J. Chem. Phys. 93, 6699 (1990).

${ }^{58}$ R. B. Williams and R. F. Loring, J. Chem. Phys. 110, 10899 (1999).

${ }^{59}$ S. Hammes-Schiffer and J. C. Tully, J. Phys. Chem. 99, 5793 (1995).

${ }^{60}$ A. Sakurai and Y. Tanimura, J. Phys. Chem. A 115, 4009 (2011).

${ }^{61} \mathrm{~K}$. Ando, "Initial value representation of the propagator for variational semiquantal wave packet" (to be published).

${ }^{62} \mathrm{~S}$. Woutersen, U. Emmerichs, H.-K. Nienhuys, and H. J. Bakker, Phys. Rev. Lett. 81, 1106 (1998).

${ }^{63}$ R. J. Abbott and D. W. Oxtoby, J. Chem. Phys. 72, 3972 (1980). 